\title{
Nonlinear homogenization techniques to solve masonry structures problems
}

\author{
Ricardo D. Quinteros ${ }^{\mathrm{a}, *}$, Sergio Oller $^{\mathrm{b}, \mathrm{c}}$, Liz G. Nallim ${ }^{\mathrm{a}}$ \\ ${ }^{a}$ Facultad de Ingeniería, ICMASa, INIQUI (CONICET), Universidad Nacional de Salta (UNSa), Avenida Bolivia 5150, 4400 Salta, Argentina \\ ${ }^{\mathrm{b}}$ CIMNE, Internacional Center for Numerical Method in Engineering, Spain \\ ${ }^{\mathrm{c}}$ UPC. Technical University of Catalonia (Barcelona Tech), Edif. C1, Campus Nord, Jordi Girona 1-3, 08034 Barcelona, Spain
}

\section{A R T I C L E I N F O}

\section{Article history:}

Available online 19 September 2011

\section{Keywords:}

Composite structures

Homogenization techniques

Masonry

Constitutive models

\begin{abstract}
A B S T R A C T
The behaviour of masonry material subjected to different in-plane loading combination is studied in this work. The masonry is considered as a periodic composite material composed by a regular distribution of brick and mortar and it is analyzed using a homogenization technique. The mechanical properties of the masonry, as an orthotropic homogeneous material, depend on the geometrical and mechanical properties of the components based on the study of the equilibrium and compatibility of a basic cell. The masonry is a frictional material and its behaviour depends on the loading direction, for these reasons, a unilateral damage model is chosen for the analysis. This model describes the behaviour of brittle materials subjected to tension-compression cyclic loads based on the introduction of two damage variables and it assumes that the damage is due to the beginning and growth of cracks only in the mortar joints. It is considered that the bricks have a linear elastic constitutive relationship. Numerical applications are performed with a nonlinear finite element code in order to test the proposed procedure by comparing the results with those available in the literature and also with experimental data.
\end{abstract}

(C) 2011 Elsevier Ltd. All rights reserved.

\section{Introduction}

Because of the heterogeneity of most building and industrial materials (mainly materials of two or more components), it is necessary to use methods and techniques to represent and reproduce the composite behaviour in both linear and nonlinear fields, either through micromodels or macromodels where the composite is considered as an homogeneous anisotropic material with average properties. An interesting alternative for the treatment of composite materials and, in particular, to analyze the behaviour of masonry is the use of homogenization techniques, which can be employed in those materials having a periodic configuration and being possible to work in two scales: a microscopic scale, which the mechanical and geometrical properties of the component materials are specified and a macroscopic scale where the material is treated as homogeneous [1]. The masonry is regarded as a composite material whose components are the bricks and the mortar used in the vertical and horizontal joints forming a periodic configuration. Thereby, it is possible, by homogenization techniques, to derive the overall behaviour of the masonry structure from the behaviour of the component materials and even adopting different constitutive models for each one.

\footnotetext{
* Corresponding author.

E-mail addresses: rdquinteros@conicet.gov.ar (R.D. Quinteros), oller@cimne. upc.edu, sergio.oller@upc.edu (S. Oller).

URLs: http://www.unsa.edu.ar, http://www.conicet.edu.ar (R.D. Quinteros), http://www.cimne.com, http://www.upc.edu (S. Oller)
}

Many authors have studied the structural behaviour of masonry. The first formulations of numerical models are based on various simplifications. These may include consideration of the masonry and its components as continuous, assuming isotropic materials and acting in the linear elastic range. Other works raise the analysis of masonry by combining structural elements of simple and known behaviours [2]. However, this does not always adequately reflect the correct and complex structural mechanism that characterizes the behaviour of the masonry.

Over the years, various models and techniques based on theoretical analysis and various levels of detail have been implemented. Among these models is, for example, the finite element method by Page [3].

This methodology allows the masonry to be modelled on a microscale, discretizing its components and analyzing in detail the local behaviour [4] or on a macroscale where it can be treated as a composite material and analyze its global behaviour. At the microscale, the bricks and the joints are represented by continuous elements, while its interface is usually represented by discrete elements. Particularly, in this work, a previously developed ad hoc homogenization proposed by Lopez et al. [5] is implemented together with the unilateral damage criteria published by Faria et al. [6].

\section{Homogenization}

An approach whose effectiveness has been demonstrated by several authors such as Lopez et al. [5], Anthoine [7], and Sacco 
[8], is to work with a combination of both scales in which there is a feedback. A periodic unit cell is identified so that its repetition generates entire masonry panel and their behaviour is analyzed as an individual problem in such a way to know the average values of the masonry as a single homogeneous material based on the actual geometrical and mechanical characteristics of constituent materials. The use of homogenization techniques saves computational work generated by the use of complex meshes, when the bricks and joints are discretized. Gabor et al. [9] presented different finite element modelling approaches for the analysis of the behaviour of unreinforced and fibre reinforced polymer strengthened masonry. In order to overcome the disadvantages of a detailed discretized modelling a homogenization of the brick/mortar assemblage was performed, obtaining a good correlation between experimental and numerical curves. On the other hand, a periodic unit cell could be used as well to perform a limit analyze for the prediction of collapse loads and homogenized failure mechanisms, such as Milani [10] and Milani et al. [11,12] modelling bricks, joints, filling resin and glass fibre reinforced polymer rods for out-of-plane loads.

\subsection{Unit cells}

The periodic structure of the masonry allows the application of homegenization techniques. It is necessary to find a unit cell or representative cell, so if this is repeated in the full dimension of the structure the original one is obtained. As it is considered that the structural element has width and height greater than the thickness,

\section{it is possible to consider a plane stress}

theplane of the masonry. When a stur tion to the plane is necessary, a three dir tative volume should be selected. It of cells, a viable and among the simp

S
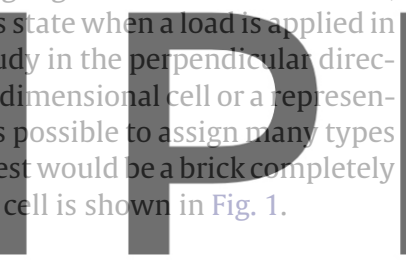

2.2. Homogeneous constitutive tensor

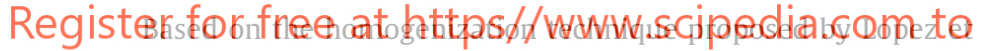

al. [5], the same deformation modes are employed to approach the compatibility and equilibrium of a unit cell shown in Fig. 2. The geometrical and mechanical properties of the components are those of the articles by Page [3] and Anthoine [7].

The different modes of deformation are depicted in Fig. 3.

Mode 1 corresponds to tension or compression stresses in $x$ direction, as shown in Fig. 3a. The equilibrium equation is given by:

$\sigma_{x} h_{C}=\sigma_{x B} h_{B}+\sigma_{x M 2} h_{M 2}=\sigma_{x M 1} h_{M 1}+\sigma_{x M 2} h_{M 2}$

$\sigma_{x}=\sigma_{x B} \frac{h_{B}}{h_{C}}+\sigma_{x M 2} \frac{h_{M 2}}{h_{C}}$

$\sigma_{x}=\sigma_{x M 1} \frac{h_{M 1}}{h_{C}}+\sigma_{x M 2} \frac{h_{M 2}}{h_{C}}$

From Fig. 2:

$\sigma_{x B} \equiv \sigma_{x M 1}$

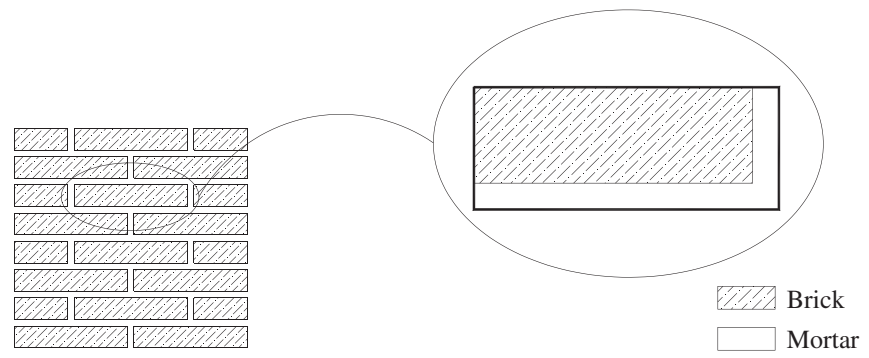

Fig. 1. Periodic structure of the masonry. Analyzed cell.

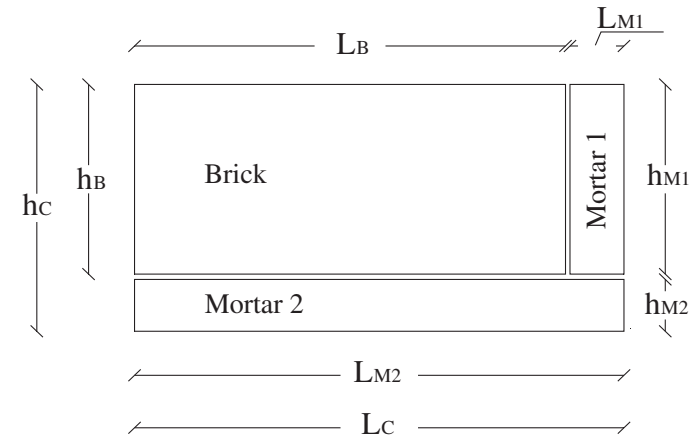

Fig. 2. Analyzed cell. Geometrical parameters.
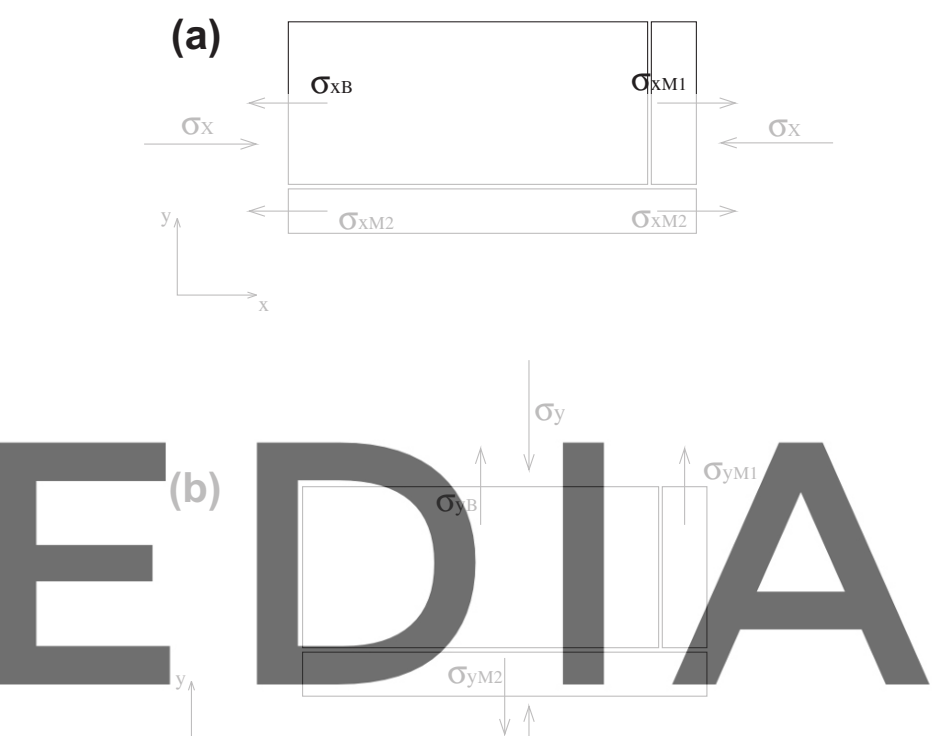

download the version without the watermark

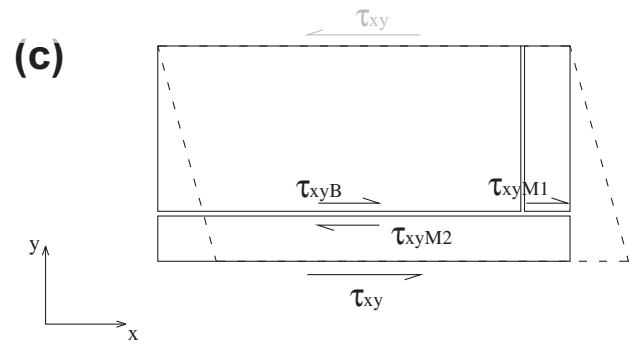

Fig. 3. Deformation modes. (a) Mode 1. (b) Mode 2. (c) Mode 3.

The compatibility equation can be written in an incremental way as follows

$\dot{\varepsilon}_{x C}=\dot{\varepsilon}_{x M 2} \frac{L_{M 2}}{L_{C}}=\dot{\varepsilon}_{x B} \frac{L_{B}}{L_{C}}+\dot{\varepsilon}_{x M 1} \frac{L_{M 1}}{L_{C}}$

The constitutive equation of the components takes into account the degradation of constitutive tensor. The masonry is a frictional material and for this reason, a damage model is chosen to represent its behaviour. It is possible to take into account the stiffness degradation due to the initiation and propagation of cracks that occurs mainly in the mortar. The model expression is:

$\dot{\sigma}_{x i}=E_{x i}(\kappa)\left(\dot{\varepsilon}_{x i}-\dot{\varepsilon}_{x i}^{p}\right)$

In Eq. (4) the Young's modulus depends on the damage parameter $\kappa$ due to the change of the elastic modulus when the elastic limit is exceeded. 
From the compatibility, equilibrium and constitutive equations is possible the determination of the stresses in the components materials as a function of the global cell stress and of its mechanical and geometrical parameters. Using Eqs. (1)-(3) the following expressions are obtained:

$\dot{\sigma}_{x B} \equiv \dot{\sigma}_{x M 1}=\dot{\sigma}_{x} A_{x B} \frac{h_{C} L_{M 2}}{E_{x M 2} h_{M 2}}=\dot{\sigma}_{x} B_{x B}$

$\dot{\sigma}_{x M 2}=\dot{\sigma}_{x} B_{x M 2}$

with:

$B_{x B}=A_{x B} \frac{h_{C} L_{M 2}}{E_{x M 2} h_{M 2}}, \quad A_{x B}=\frac{1}{\left(\frac{L_{B}}{E_{x B}}+\frac{L_{M 1}}{E_{x M 1}}+\frac{h_{B} L_{M 2}}{E_{x M 2} h_{M 2}}\right)}, \quad B_{x M 2}=\frac{h_{C}-B_{x B} h_{B}}{h_{M 2}}$

Finally, employing the compatibility Eq. (3), the constitutive Eq. (4) and considering $\sigma_{x B} \equiv \sigma_{x M 1}$ (Eq. (2)), the cell strain can be written as:

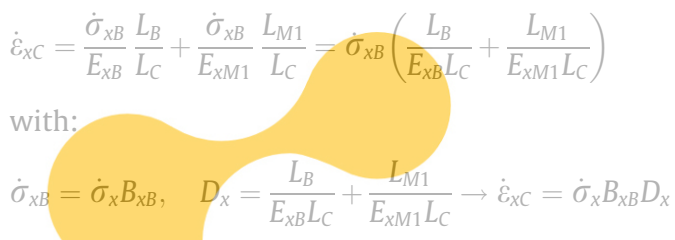

Then, the homogenized value in $x$ direction for the constitutive tensor can be derived from Eq. (7) as:

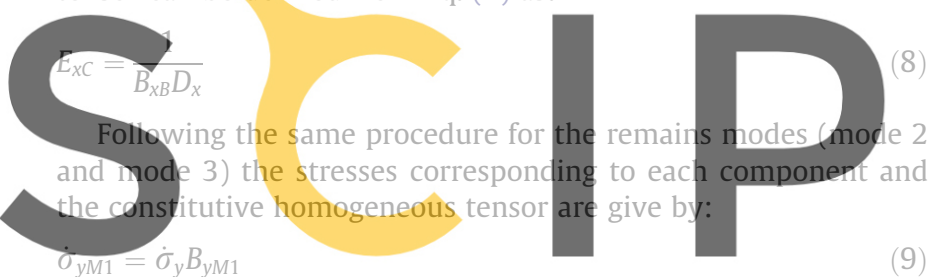

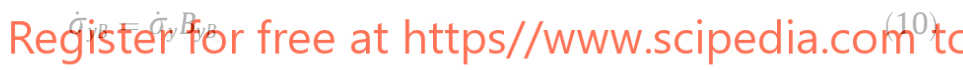

\section{$\dot{\sigma}_{y \mathrm{M} 2}=\dot{\sigma}_{y}$}

with,

$B_{y M 1}=A_{y M 1} \frac{L_{C} h_{B}}{E_{y B} L_{B}}, \quad A_{y M 1}=\frac{1}{\left(\frac{L_{M 1} h_{B}}{E_{y M 1} L_{B}}+\frac{h_{M 1}}{E_{y M 1}}\right)}, \quad B_{y B}=\frac{L_{C}-B_{y M 1} L_{M 1}}{L_{B}}$

From compatibility equations and equilibrium equation for mode 2:

$\dot{\varepsilon}_{y C}=\dot{\sigma}_{y}\left(\frac{h_{M 2}}{h_{C} E_{y M 2}}+\frac{B_{y B} h_{B}}{E_{y B} h_{C}}\right)$

Finally, from Eq. (12):

$$
E_{y C}=\frac{1}{\frac{h_{M 2}}{h_{C} E_{y M 2}}+\frac{B_{y B} h_{B}}{E_{y B} h_{C}}}
$$

Meanwhile from the representation of mode 3:

$$
\begin{aligned}
& \dot{\tau}_{B}=\dot{\tau} A_{x y B} \\
& \dot{\tau}_{M 1}=\dot{\tau} A_{x y M 1} \\
& \dot{\tau}_{M 2}=\dot{\tau}
\end{aligned}
$$

with,

$A_{x y B}=\frac{G_{x y B} L_{C}}{G_{x y M 1} L_{M 1}+G_{x y B} L_{B}}, \quad A_{x y M 1}=\left(\frac{L_{C}}{L_{M 1}}-A_{x y B} \frac{L_{B}}{L_{M 1}}\right)$

From compatibility equations and equilibrium equation for mode 3:

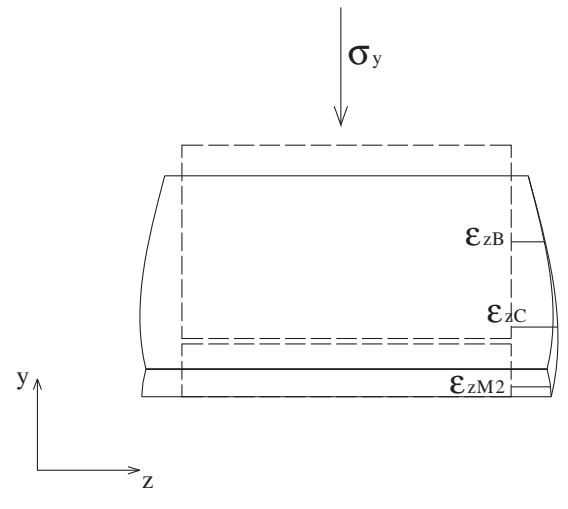

Fig. 4. Deformation mode 4.

$\dot{\gamma}_{x y C}=\dot{\tau}\left(\frac{h_{M 2}}{G_{x y M 2} h_{C}}+\frac{A_{x y B} h_{B}}{G_{x y B} h_{C}}\right)$

Finally, from Eq. (17):

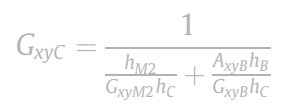

Also, it is possible to analyze a mode 4 , which corresponds to out of plane strain. However, in this work only cases of plane stresses are considered and thereby the strain in $x z$ plane is limited to that produced by Poisson effect. Obviously, the strain has a discon-
tinuity in the zone where the brick is supported by the mortar due
to the strong difference betheen their properties. The homoge-
nized strain $\varepsilon_{z C}$ of the cell in $z$ direction is considered as the envel-
opment of the cornponents deformation curves (Fig. 4 ).
Taking as starting point the expression of the Secant Constitu-
tive Matrix in the case of orthotropy, we can obtain fleformation

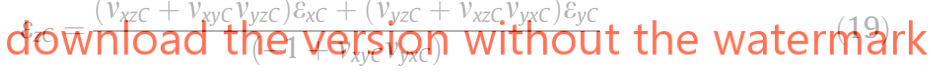

\section{3. impiemented models}

As mentioned, the use of homogenization for the resolution of structural problems, through the interaction of the microscale and macro-scale and feedback, allows separating the behaviour of the component materials each one governed by different constitutive models.

For the bricks a linear elastic constitutive law is considered. The stress-strain relationship can be written as:

$\sigma_{B}=C_{B} \varepsilon_{B}$

with

$\sigma_{B}=\left(\sigma_{x B}, \sigma_{y B}, \tau_{x y B}\right)^{T}$ and $\varepsilon_{B}=\left(\varepsilon_{x B}, \varepsilon_{y B}, \gamma_{x y B}\right)^{T}$

To characterize the behaviour of masonry as a frictional material and to distinguish the behaviour under compression and tension loads to the mortar a unilateral damage model is implemented [6]. The most important feature of this model is the fact that damage is irreversible but can be active or not depending on load conditions. The mortar microcracks can occur due to tension loads, but them can be "sealed" if the load direction changes. Thus, the material can regain its initial stiffness in this case the damage is present but is considered inactive.

For the description of the model is necessary to define two scalar damage variables, $d^{+}$and $d^{-}$, for tension and compression stresses respectively. The effective stress is split into a positive part $\sigma_{i j}^{+}$ and a negative $\sigma_{i j}^{-}$, being: 
$\bar{\sigma}_{i j}=\bar{\sigma}_{i j}^{+}+\bar{\sigma}_{i j}^{-}$

where:

$\bar{\sigma}_{k l}^{+}=\sum_{i}\left\langle\bar{\sigma}_{i}\right\rangle p_{k}^{i} p_{l}^{i}$

with $\sigma_{i}$ : main stresses, $p_{k}^{i}$ : principal directions, $\langle\cdot\rangle$ : ramp function.

The Helmholtz free energy is given by:

$\Psi\left(\varepsilon, d^{+}, d^{-}\right)=\left(1-d^{+}\right) \Psi_{0}^{+}(\varepsilon)+\left(1-d^{-}\right) \Psi_{0}^{-}(\varepsilon)$

where $\Psi_{0}^{+}$and $\Psi_{0}^{-}$are given by:

$\Psi_{0}^{+}=\frac{1}{2} \bar{\sigma}_{i j}^{+} C_{i j k l}^{o}{ }^{-1} \bar{\sigma}_{k l}=\frac{1}{2} \bar{\sigma}_{i j}^{+} \varepsilon_{i j}, \quad \Psi_{0}^{-}=\frac{1}{2} \bar{\sigma}_{i j}^{-} C_{i j k l}^{o}{ }^{-1} \bar{\sigma}_{k l}=\frac{1}{2} \bar{\sigma}_{i j}^{-} \varepsilon_{i j}$

\subsection{Damage criterion}

The damage criterion used in this work is that from Faria et al. [6] who considered it similar to scalar damage criteria by Simo and Ju [13], but distinct in each direction, so an equivalent tension stress and compressive stress are defined equivalent and that is briefly described here:
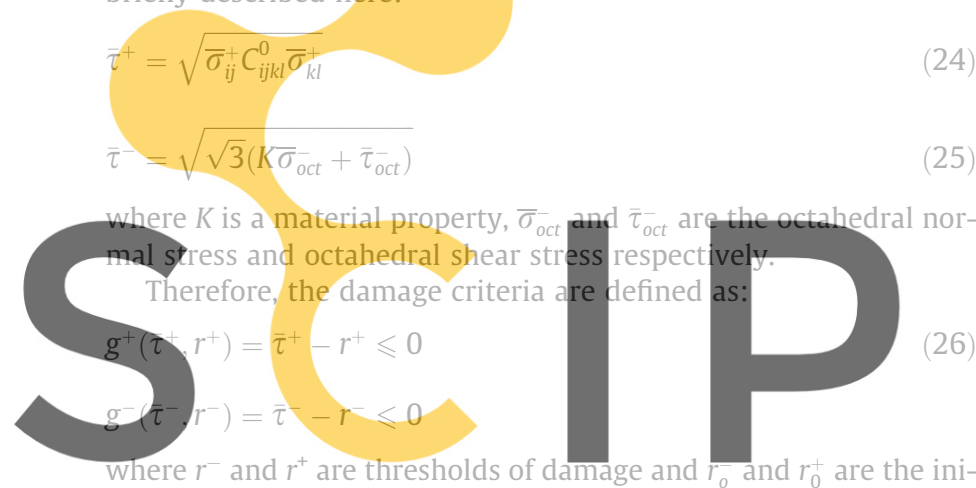
tial parameters of damage:

\section{Register for free at https//www.scipedia.com to
\[ r_{0}^{+}=\frac{\sigma}{\sqrt{E_{0}}}, \quad r_{0}^{-}=\sqrt{\frac{\sqrt{3}}{3}}(k-\sqrt{2}) \bar{\sigma}^{-} \]}

The evolution laws of internal variable $d^{+}$is given by

$$
\begin{aligned}
& \dot{d}^{+}=\dot{\vartheta}^{+} \frac{\partial G^{+}\left(r^{+}\right)}{\partial r^{+}} \\
& \dot{r}^{+}=\dot{\vartheta}^{+}(\geqslant 0)
\end{aligned}
$$

where $G^{+}$is an arbitrary monotonic increasing function and $\vartheta^{+}$is the damage parameter of consistency. The evolution law of $d^{-}$is the same as the previous for the other direction but with $G^{-}$and $\vartheta^{-}$ respectively.

The conditions of loading and unloading of Kuhn-Tucker are expressed as:

$\dot{\vartheta}^{+} \geqslant 0, \quad g^{+} \leqslant 0, \quad \dot{\vartheta}^{+} g^{+}=0$

For a given generic time is

$$
r^{+}=\max \left\{r_{0}^{+}, \max _{s \in[0, t]}\left(\bar{\tau}_{s}^{+}\right)\right\} \quad \text { and } \quad r^{-}=\max \left\{r_{0}^{-}, \max _{s \in[0, t]}\left(\bar{\tau}_{s}^{-}\right)\right\}
$$

The rule of evolution of variables $d^{+}$and $d^{-}$have been derived using as a basis previous works. In particular for $d^{+}$, the adopted law is that from Oliver et al. [14]:

$d^{+}=G^{+}\left(r^{+}\right)=1-\frac{r_{0}^{+}}{r^{+}} e^{A^{+}\left(1-\frac{r^{+}}{r_{0}^{+}}\right)}$

$$
A^{+}=\left(\frac{G_{f} E}{l_{C H} r_{0}^{+2}}-\frac{1}{2}\right)^{-1} \geqslant 0
$$

where $G_{f}$ is the tensile fracture energy and $l_{C H}$ is the "characteristic length", depending on the size of the element adopted for the spatial discretization (Oliver [15])

For the variable $d^{-}$, the law of evolution is adopted from Mazars

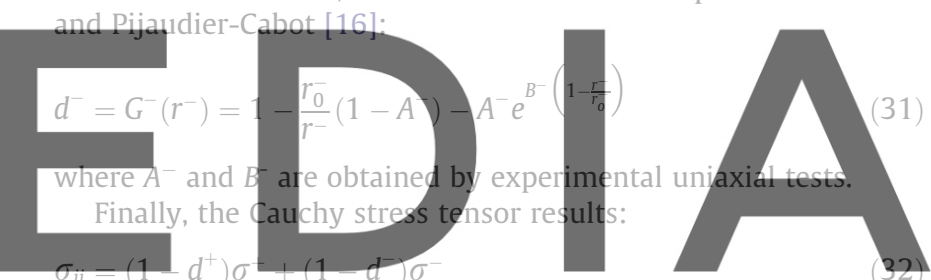

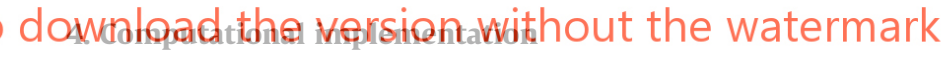

The described model has been implemented in a global finite element code (FEM) PLCD4.02 [17]. This way, it is possible to solve the individual problem in each scale and the feedback between them. In the microscale must be differentiated the constitutive model for each component and the macroscale which solves the composite structure as a single homogeneous material whose mechanical properties are updated at each load step according to

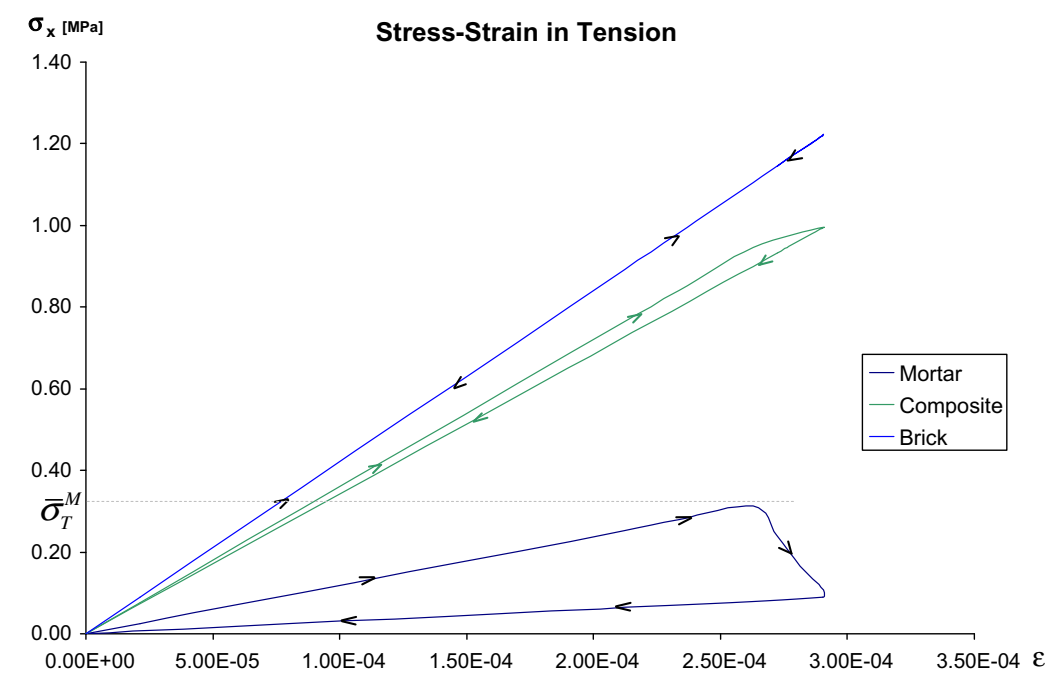

Fig. 5. Behaviour of the composite and its components. 
what happened with the brick and mortar, if the latter exceeds, or not, its elastic limit.

\section{Numerical results}

To show the behaviour of the composite and its individual components, a simple uniaxial test with cyclic loads is performed. The results depict the typical performances of the constitutive model used for the mortar during tension-compression cyclic loads. A complex loading scheme has been imposed, comprising an incursion into tensile regimen up to the initial elastic threshold, and leading to damage thereafter. Loading is then reversed, producing a return to the initial state and a subsequent incursion into compression up to its initial elastic threshold, also the stiffness is recovered. From there, progressive damage deformation is observed; then a new load reversal is enforced. This unilateral effect corresponds to a peculiar feature of brittle materials behaviour, fully captured by the proposed model, owing to its "memory" proficiency. The curve in Fig. 5, also shows the composite stiffness degradation due to the mortar behaviour, which is showed in Fig. 6 for the complete tension-compression cyclic load.

To analyze the behaviour of the masonry with the implemented model in the finite element code, the following brick properties are used:

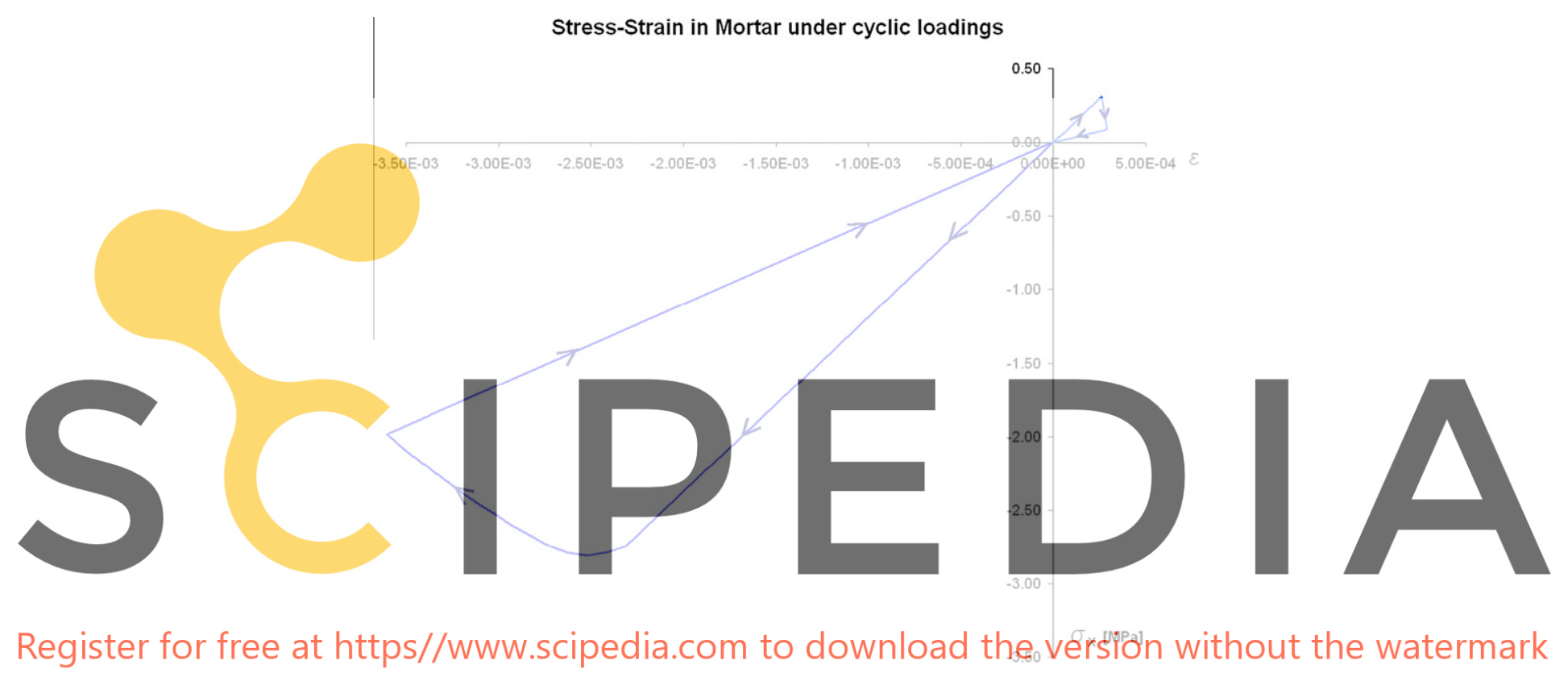

Fig. 6. Behaviour of the mortar under tension-compression cyclic load.

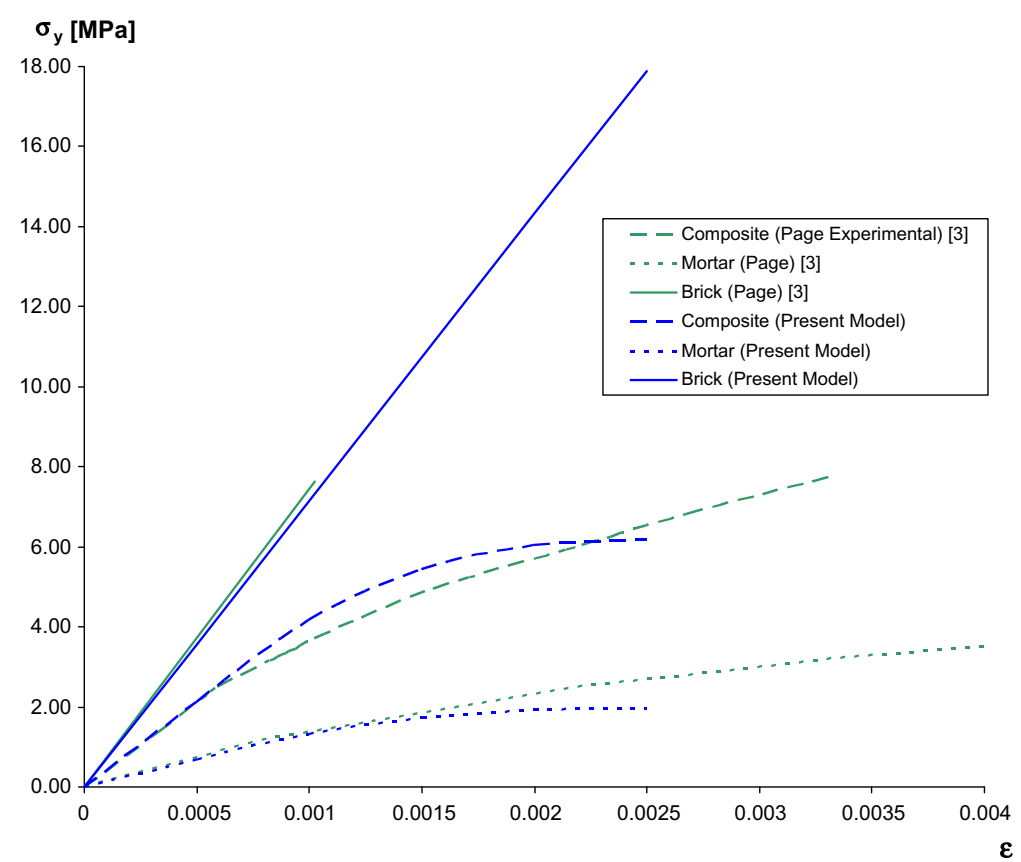

Fig. 7. Comparisons of stress-strain curves for composite and components. 
$E_{x}=5900 \mathrm{MPa}, E_{y}=7550 \mathrm{MPa}, E_{z}=5900 \mathrm{MPa}$, Poisson coefficient $v=0.167$. Meanwhile, for the mortar an isotropic constitutive tensor is chosen, with: $E=1200 \mathrm{MPa}$ and Poisson coefficient $v=0.21$.

A masonry panel, studied experimentally by Page [3] is chosen for comparison purposes. This test is one of the most commonly used in the calibration of numerical models of masonry. Masonry panels of $50 \times 24 \times 5.4 \mathrm{~cm}^{3}$ were tested in uniaxial compression with load applied normal to the bed joint, stress-strain values were measured on a central gage length for the composite, and the curves for the bricks and mortar has been adopted by Page in an analytical way. The marked difference in stiffness between the composites is apparent, but the mortar does not have a limit. In the present model, the mortar reaches a limit value $\bar{\sigma}_{C}^{M}=0.32 \mathrm{MPa}$ and has no capacity to take more stress. The obtained results and the comparisons with Page [3] are shown in Fig. 7.

Luccioni and Martin [18] also used the Page test for calibrating their model, which are also compared with the results of the model proposed here (Fig. 8).

The panel is considered under a compression load parallel and perpendicular to the mortar joint and the resulting curves forcevertical and horizontal displacement are plotted in Fig. 8. In the case of the horizontal compression load, since no experimental data by Page was available, the author made a general and a fine mortar-brick discretization. A very close agreement is found as the result of the comparison.

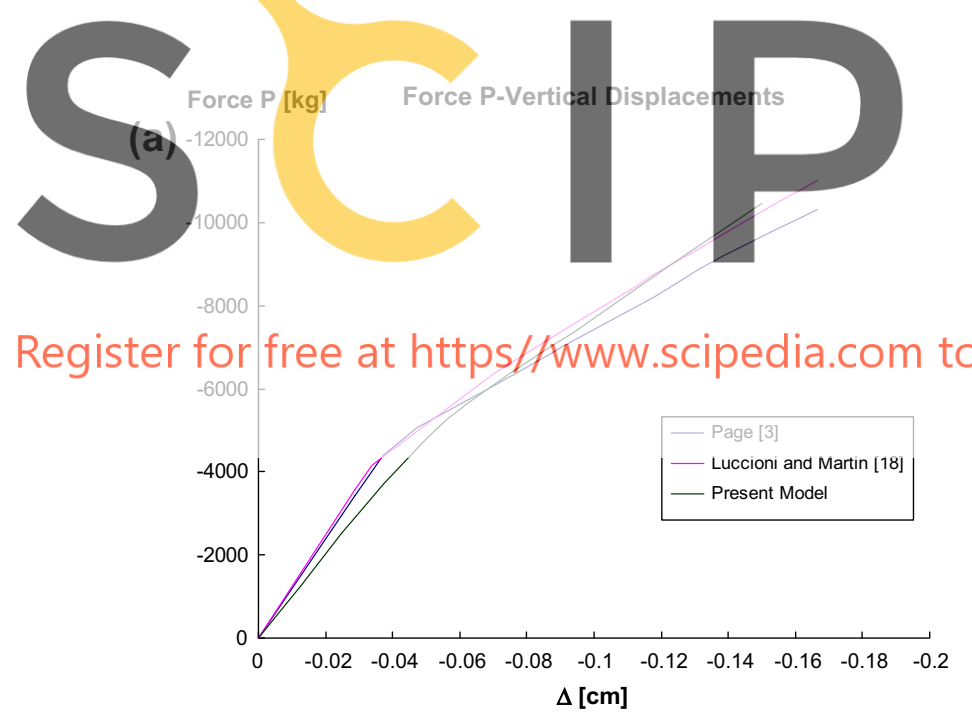

\section{Force P [kg] Force P-Horizontal Displacement}

(b)

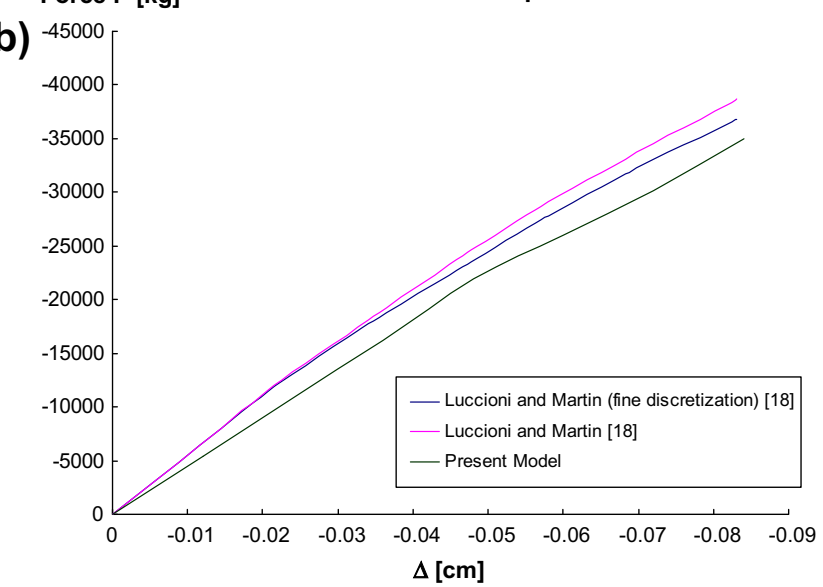

Fig. 8. Force-vertical (a) and horizontal (b) displacement for a masonry panel.

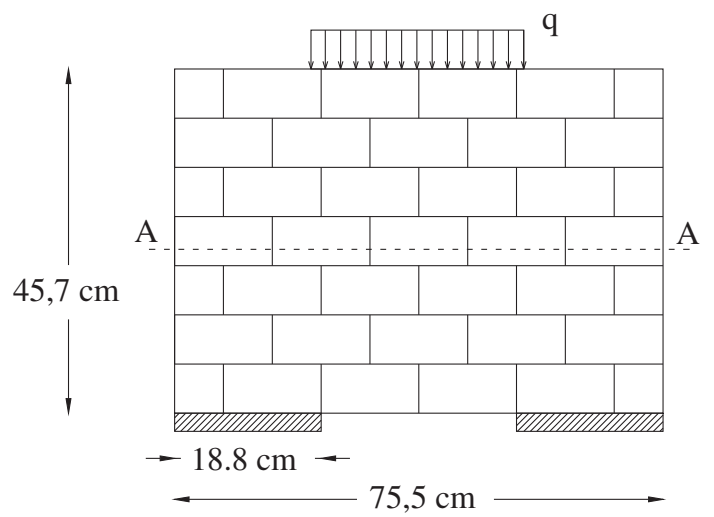

Fig. 9. General arrangement of the simulated masonry bending test by Page [3].

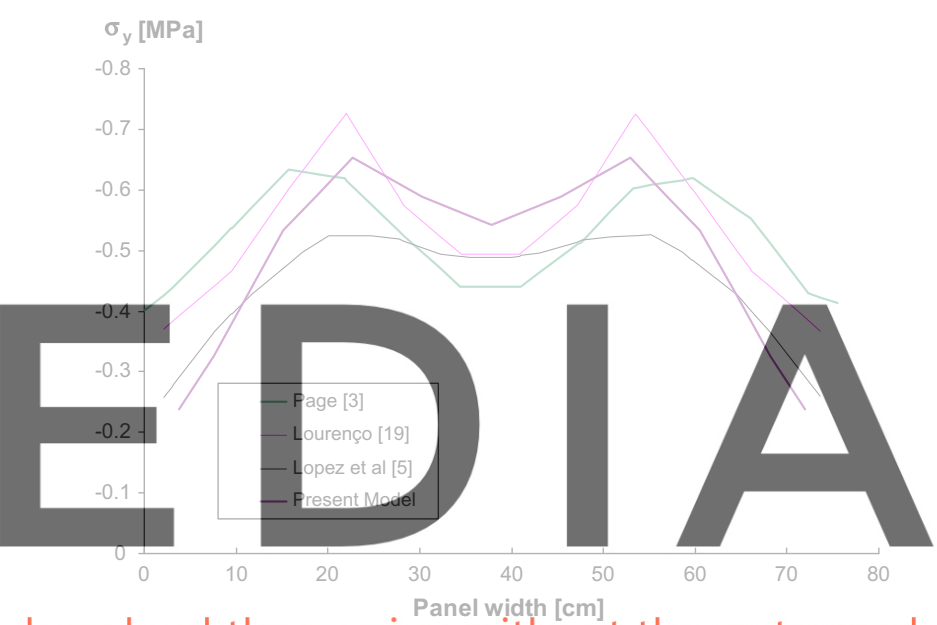

download the version without the watermark Fig. 10. Stress-strain for $20 \mathrm{kN}$.

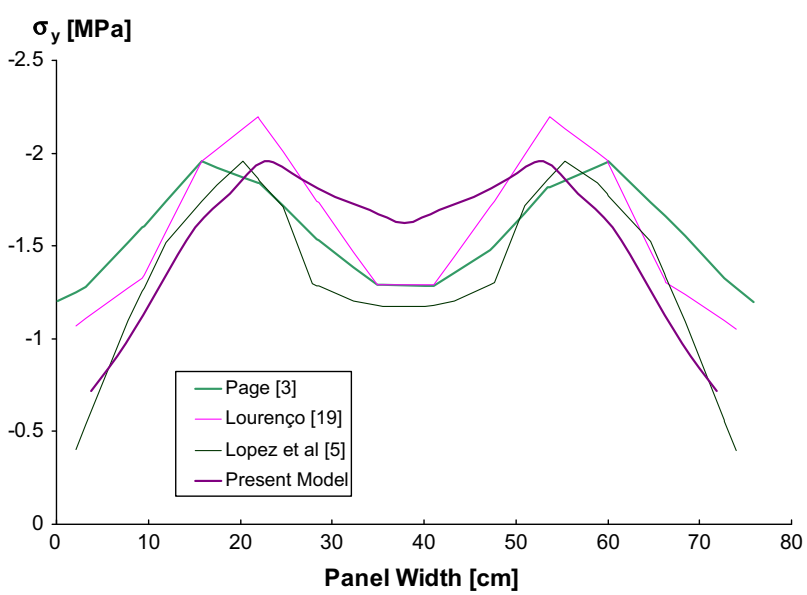

Fig. 11. Stress-strain for $60 \mathrm{kN}$.

A bending test on a panel did by Page [3] has been also simulated with a mesh of 100 four-node elements. The testing arrangement is shown in Fig. 9, and the mechanical properties of the materials are the same as before and fracture energies $G_{f}^{M}=0.16 \mathrm{~kg} / \mathrm{cm}$ and $G_{f}^{B}=6 \mathrm{~kg} / \mathrm{cm}$ have been assumed for the 
mortar and the bricks respectively. Vertical strain on level A-A was measured, then vertical stress distribution along A-A was determined. The comparison was performed for the loads of 20 and $60 \mathrm{kN}$, and the results obtained are given in Figs. 10 and 11. Models by Lourenço [19] and Lopez [5] were compared as well.

\section{Conclusions}

A methodology using homogenization techniques to solve masonry problems subjected to in-plane loadings has been developed in this work. The masonry is considered as a homogeneous material due to its periodic structure made up of mortar and bricks with different mechanical and geometrical characteristics. The ad hoc homogenized model allows a simplified treatment of the masonry. The usefulness of this type of homogenization is the capability to save on computational time and to simplify the mesh generation process when the model is implemented in a global finite element code. The number of elements needed is much smaller than in macromodels.

A linear elastic constitutive law is considered for the bricks, while the unilateral damage model is implemented for the mortar in order to describe the behaviour of brittle materials subjected to alternating tension-compression cyclic loads, based on the introduction of two scalar damage variables for tension and compression stresses respectively.

The heterogeneity in the composition of the masonry along with the arrangement of the elements (bricks and joints) lead to

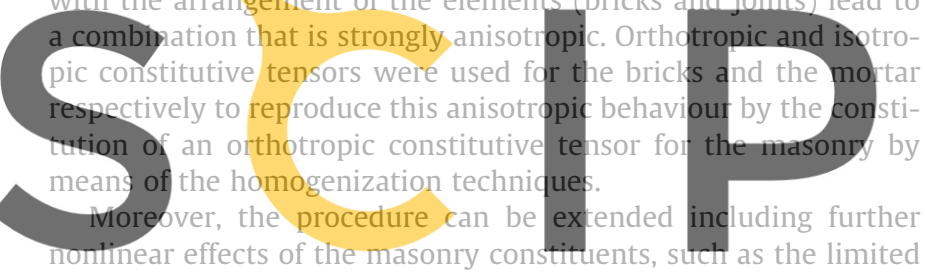
compressive strength and the tensile failure of the bricks and to

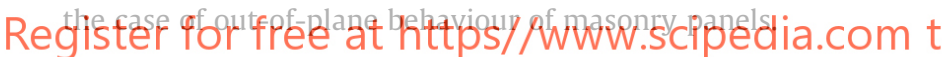

\section{References}

[1] Oller S. Análisis y cálculo de estructuras de materiales compuestos. Centro Internacional de Métodos Numéricos en Ingeniería (CIMNE). Barcelona, España; 2002.

[2] Roca P, Molins C, Marí AR. Strength capacity of masonry wall structures by the equivalent frame method. J Struct Eng 2005;131(10):1601-10.

[3] Page AW. Finite element model for masonry. J Struct Div 1978;104(ST8):1267-85 [ASCE].

[4] Zucchini A, Lourenço PB. A micro-mechanical model for the homogenisation of masonry. Int J Solids Struct 2002;39:3233-55.

[5] Lopez J, Oller S, Oñate E, Lubliner J. A homogeneous constitutive model for masonry. Int J Numer Methods Eng 1999;46:1651-71.

[6] Faria R, Oliver J, Cervera M. A strain-based plastic viscous-damage model for massive concrete structures. Int J Solids Struct 1998;35(14):1533-58.

[7] Anthoine A. Derivation of the in-plane elastic characteristics of masonry through homogeneization theory. Int J Solids Struct 1995;32(2):137-63.

[8] Sacco E. A nonlinear homogenization procedure for periodic masonry. Eur J Mech A/Solids 2009;28:209-22.

[9] Gabor A, Bennani A, Jacquelin E, Lebon F. Modelling approaches of the in-plane shear behaviour of unreinforced and FRP strengthened masonry panels. Compos Struct 2006;74(3):277-88.

[10] Milani G. FE homogenized limit analysis model for masonry strengthened by near surface bed joint FRP bars. Compos Struct 2010:92(2):330-8.

[11] Milani G, Bucchi A. Kinematic FE homogenized limit analysis model for masonry curved structures strengthened by near surface mounted FRP bars. Compos Struct 2010:93(1):239-58

[12] Milani G, Milani E, Tralli A. Approximate limit analysis of full scale FRPreinforced masonry buildings through a 3D homogenized FE package. Compos Struct 2010;92(4):918-35.

[13] Simo C, Ju J. Stress and strain based continuum damage Models:I. formulation. Int J Solids Struct 1987;23:821-40

[14] Oliver J, Cervera M, Oller S, Lubliner J. Isotropic damage models and smeared crack analysis of concrete. In: Proceedings of the 2 nd international conference on computer aided, analysis and design of concrete structures. Austria: Zell am

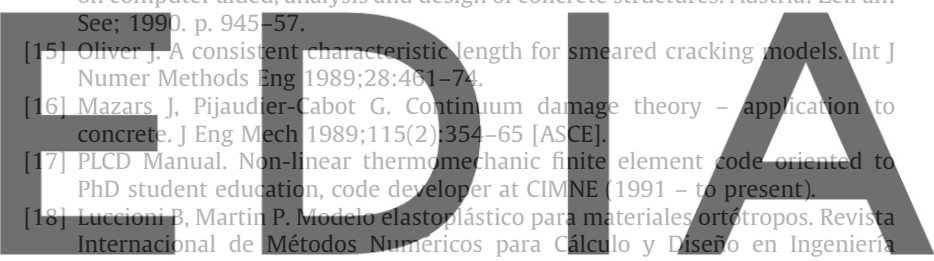
$1997: 13(4): 603-14$

[19] Lourenço PB. Computational strategies for masonry structures. Doctoral thesis,

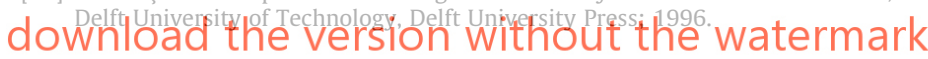

